\title{
Habilidades motrices básicas en los fundamentos técnicos del fútbol en niños de instituciones educativas de primaria, Puno.
}

\author{
Percy Bustinza Mendizábal \\ Universidad Cesar Vallejo \\ pbusmen@ucvvirtual.edu.pe \\ https://orcid.org/0000-0001-5355-2958 \\ Dulio Oseda Gago \\ Universidad Cesar Vallejo \\ dodedag@ucvvirtual.edu.pe \\ https://orcid.org/0000-0002-3136-6094
}

\section{RESUMEN}

Con el presente trabajo de Investigación se canalizó aportes orientados para la mejora de las habilidades motoras y la práctica del fútbol, en los niños de la región Puno y el resto del país. El objetivo general es determinar de qué manera las habilidades motrices básicas influirán en los fundamentos técnicos del fútbol en niños de 09 y 11 años en las instituciones educativas de educación primaria de Puno, en el año 2019. En la investigación se utilizó el diseño correlacional causal, el tipo de investigación es básica o pura, la población está constituida por 537 estudiantes y la muestra conformada por 81 estudiantes de instituciones educativas de primaria Puno, para la recolección de datos se elaboraron fichas de observación los cuales corresponden a un trabajo de campo donde se miden las habilidades motrices básicas y fundamentos técnicos del fútbol. Con los resultados de la prueba de hipótesis general haciendo uso de la correlación de Pearson podemos determinar que las habilidades motrices básicas influyen en los fundamentos técnicos del fútbol porque los resultados según el SPSS igual a $0.681^{* *}$ nos indica que existe una correlación altamente significativa entre las habilidades motrices básicas y los fundamentos técnicos de fútbol en niños de 09 y 11 años de las instituciones educativas de educación primaria de Puno, en el año 2019. Dichos hallazgos nos permiten concluir que las habilidades motrices tienen relevancia y mejoran progresivamente el nivel de los fundamentos técnicos del fútbol en las instituciones mencionadas.

Palabras Clave: Habilidad, fundamentos, manipulación. 


\title{
Basic motor skills in the technical foundations of soccer in children from elemental educational institutions, Puno.
}

\begin{abstract}
With this research work, contributions aimed at improving motor skills and playing soccer were channeled in children from the Puno region and the rest of the country. The general objective is to determine how basic motor skills will influence the technical foundations of soccer in children aged 09 and 11 years in the educational institutions of elementary education in Puno, in 2019. The research used the causal correlational design, the type of research is basic or pure, the population is made up of 537 students and the sample made up of 81 students from Puno elementary educational institutions, for data collection observation files were prepared which correspond to a field work where basic motor skills and technical foundations of soccer are measured. With the results of the general hypothesis test using Pearson's correlation, we can determine that basic motor skills influence the technical foundations of soccer because the results according to the SPSS equal to $0.681 * *$ indicate that there is a highly significant correlation between the basic motor skills and the technical foundations of soccer in children aged 9 and 11 from the educational institutions of elementary education in Puno, in 2019. These findings allow us to conclude that motor skills are relevant and progressively improve the level of technical foundations of soccer in the aforementioned institutions.
\end{abstract}

Key words: Skill, fundamentals, manipulation.

Artículo recibido: 10. Mayo. 2021

Aceptado para publicación: 28. junio. 2021

Correspondencia: pbusmen@ucvvirtual.edu.pe

Conflictos de Interés: Ninguna que declarar 


\section{INTRODUCCIÓN}

El estudio se orienta a dar respuestas a la problemática de las habilidades motrices y fundamentos del fútbol los cuales se plasman en estudios realizados a nivel internacional en países como España, Alemania, Inglaterra, Francia, en sus investigaciones concluyen que estas variables van enlazadas, es decir que una es consecuencia de la otra. (Alonso, 2020; Costa Riveiro 2018) en el contexto sudamericano presentan buena organización y planificación a largo plazo países como Brasil, Argentina, Colombia en lo que respecta al futbol en categoría de menores. A nivel nacional son pocas las investigaciones referentes a estas teorías, no existe planificación a largo plazo como ocurre a nivel internacional, estos postulados nos motivan para realizar cambios a nivel de estado, MINEDU, IPD, gobiernos regionales, gobiernos locales, los cuales deben invertir en campos deportivos de fútbol, infraestructura deportiva en instituciones del nivel primaria, materiales deportivos, capacitación y becas para docentes y entrenadores de fútbol. En las instituciones educativas primarias de Puno los estudiantes presentan deficiencias en sus habilidades motrices y dominio de las capacidades técnicas del fútbol, razón por la cual con el presente artículo científico hemos contribuido para que estas condiciones cambien porque demostramos que con trabajo, planificación y disciplina los niños mejoran sus condiciones técnicas y su desenvolvimiento en el campo de juego, resultados que permiten realizar ajustes a nivel de docentes de educación física y entrenadores, los cuales van tomando conciencia acerca de la importancia sobre su correcta aplicación de los fundamentos técnicos del fútbol en la categoría de menores Peraza (2020). Analizando esta problemática es pertinente comprobar que el niño al practicar las habilidades motrices básicas en sus dimensiones locomotrices, manipulativas y de estabilidad mejora progresivamente el aprendizaje de las habilidades motrices específicas Fort, (2016). Por consiguiente, el niño evoluciona y mejora sus condiciones en la práctica del fútbol. En el contexto social estamos seguros de contribuir que los niños del nivel primario a través de su interacción, en sociedad estos mejoran sus habilidades motrices y específicas, estas condiciones se dan mediante las actividades lúdicas y el juego. (Castañer y Camerino 1991; Gallahue 2006).

Descrito la problemática de la investigación, permite plantear el problema general: ¿De qué manera las habilidades motrices básicas influyen en los fundamentos técnicos del fútbol en niños de 09 y 11 años en las instituciones educativas de educación primaria de Puno, en el año 2019?, Problemas Específicos: ¿De qué manera las habilidades motrices básicas influyen en 
los fundamentos técnicos del fútbol con balón en niños de 09 y 11 años en las instituciones educativas de educación primaria de Puno, en el año 2019? ¿De qué manera las habilidades motrices básicas influyen en los fundamentos técnicos del fútbol sin balón en niños de $09 \mathrm{y}$ 11 años en las instituciones educativas de educación primaria de Puno, en el año 2019?, la investigación resalta el objetivo general denominado determinar de qué manera las habilidades motrices básicas influyen en los fundamentos técnicos del fútbol en niños de 09 y 11 años en las instituciones educativas de educación primaria de Puno, en el año 2019. Del mismo modo mencionamos objetivos específicos Determinar de qué manera las habilidades motrices básicas influyen en los fundamentos técnicos del fútbol con balón en niños de 09 y 11 años en las instituciones educativas de educación primaria de Puno, en el año 2019. Determinar de qué manera las habilidades motrices básicas influyen en los fundamentos técnicos del fútbol sin balón en niños de 09 y 11 años en las instituciones educativas de educación primaria de Puno, en el año 2019. Además, resaltamos la hipótesis general. Las habilidades motrices básicas influyen significativamente en los fundamentos técnicos del fútbol en niños de 09 y 11 años en las instituciones educativas de educación primaria de Puno, en el año 2020. Las habilidades motrices básicas influyen significativamente en los fundamentos técnicos de fútbol con balón en niños de 09 y 11 años en las instituciones educativas de educación primaria de Puno, en el año 2020. Las habilidades motrices básicas influyen significativamente en los fundamentos técnicos de fútbol sin balón en niños de 09 y 11 años en las instituciones educativas de educación primaria de Puno, en el año 2020.

El presente estudio se fundamenta en el aspecto social, porque es imprescindible que los niños desarrollen sus habilidades motoras, interactuando en sociedad, en grupos, en su contexto social, el mismo que ayuda al niño a lograr una mayor adquisición motriz, para luego plasmarlo en los deportes. Implicancias prácticas: al poner en práctica las habilidades motrices básicas se fortalece la coordinación motriz en los estudiantes agrupados en los dominios: locomoción, manipulación y estabilidad, del mismo modo los fundamentos técnicos del fútbol agrupados en los dominios: fundamentos técnicos con balón y fundamentos técnicos sin balón, con el transcurrir de los años el niño va perfeccionando estas habilidades. Aportamos valor teórico respecto a las variables en estudio: habilidades motrices básicas y fundamentos técnicos del fútbol con balón y sin balón. Expresamos utilidad metodológica, porque elaboramos cuestionarios para recolectar información de primera mano, los cuales serán validados por doctores en educación, además estos 
instrumentos y diseño servirán como guía para otros investigadores, estudiantes y profesionales del área de educación física.

Fort, (2016) ¿Por qué es importante desarrollar la competencia motriz en la infancia y la adolescencia? Base para un estilo de vida saludable. Universidad de Girona, Salt, Barcelona. España. La investigación considera la importancia de las habilidades motrices en infantes, niños, adolescentes y como estos mejoran progresivamente sus habilidades específicas según su edad. Razón por la cual el niño desde los 7 a 11 años mejora sus habilidades motrices específicas, en este caso los adapta a la práctica del fútbol, además de su mejora progresiva de sus capacidades físicas según su edad.

Costa Riveiro, (2018) Desempeño motor en habilidades básicas de niños participantes y no participantes de práctica deportiva orientada. Universidad Federal de Minas Gerais, Belo Horizonte-MG, Brasil. Podemos concluir que el contexto de práctica deportiva orientada ha mostrado una manera de influir positivamente en el desarrollo motor de las habilidades básicas de los niños, contribuyendo en general al proceso de adquisición y refinamiento de las habilidades motoras básicas, contrariamente en nuestra región de Puno y el resto del país incluyendo la capital es escaso el tiempo que se le brinda a nuestros niños, su práctica solo se reduce a las clases de educación física, en la Región de Puno el niño mejora sus habilidades sin darse cuenta, es decir en su comunidad, en familia, con sus amigos. Por consiguiente, a nivel de estado se debe incrementar las horas de educación física.

Jakub Kookstejn, Martin Musalek (2019) La relación entre las habilidades motoras fundamentales y las habilidades específicas del juego en jóvenes futbolistas de élite. University, CZECH REPUBLIC. En los jugadores se encontró que el salto horizontal y la captura son predictores especialmente significativos de GSMS. Basado en estos resultados, los entrenadores de jugadores juveniles deben enfatizar el desarrollo suficiente de FMS, particularmente durante la niñez media. Los futuros investigadores deberían determinar si esta relación es cierta en un tamaño de muestra mayor, y si existen diferencias en FMS entre los jugadores de fútbol de élite y sub-élite.

Solorzano, (2017), Las capacidades coordinativas y los fundamentos técnicos del fútbol en la categoría sub 12 de la institución educativa Nº 21544 La Villa en el año 2017. Universidad Nacional José Faustino Sanchez Carrión. Huacho Lima. Se concluyo que las capacidades coordinativas influyen en los fundamentos técnicos del fútbol. Pero requiere trabajar la conducción porque presentan un nivel regular o malo en un $56 \%$, en el dribleo al $47 \%$ le 
falta mejorar. La precisión en el remate está en un 57\% es decir esta entre regular y malo. Se recomienda que la población y muestra de la investigación debió abarcar más instituciones educativas porque los resultados son pobres cuando se trata de una sola institución, además se debe tamizar el grupo, es decir seleccionar a los estudiantes con mejores condiciones técnicas para la práctica del fútbol, analizando las 2 variables de este estudio son similares a nuestra propuesta, pero consideramos que la nuestra es mejor porque abarca a todas las instituciones educativas primarias de Puno cercado.

Garay y Romero (2012), en su tesis. El juego como medio didáctico en el desarrollo de las habilidades motrices básicas de los alumnos del IV ciclo del nivel primario de la I.E.P. "La edad de oro". Universidad Nacional José Faustino Sánchez Carrión de Huacho. Lima. Concluye que tiene relación directa entre la expresión corporal y las habilidades motrices básicas, además, está demostrado a través de múltiples investigaciones que el niño jugando con sus pares en sociedad adquiere mejoras en sus habilidades motrices. El docente se convierte en su guía, en su mediador de sus aprendizajes y su labor es fundamental en el desarrollo de sus habilidades específicas, es decir en su nivel progresivo de sus capacidades técnicas en la práctica del fútbol.

Las variables habilidades motrices básicas y fundamentos técnicos del fútbol, los ubicamos en el enfoque epistemológico desde el empirismo idealista porque esta investigación está basada en la experiencia y observación, es decir el niño aprende a través de la observación, de la imitación, mediante juegos, por eso es importante el uso de materiales didácticos, a través de métodos activos innovadores y este poco a poco adquiere experiencia en su ejecución de destrezas motoras específicas, es decir lo adapta a los diversos deportes, en este caso el fútbol. Asimismo, esta teoría está basada en el enfoque epistemológico de el positivismo, tomando como referencia el estadio del conocimiento positivo, en el cual se aplica métodos modernos, como la observación, la experimentación, razón por la cual el niño experimenta cambios en su adaptación al deporte del fútbol, valora sus capacidades técnicas, capacidades físicas y posteriormente sus capacidades tácticas, este a través de la práctica mejora su conducción, el dribling, el remate, su recepción con los distintos segmentos del cuerpo, su técnica correcta del cabeceo, mejora su coordinación en la carrera y los saltos, es decir es imprescindible lograr en ellos su autonomía, su deseo de superación Ñaupas, (2018). El principio psicológico es fundamental en su aprendizaje, es decir debe tener enriquecido su espíritu volitivo, el cual significa que tenga ganas de aprender, ganas de lograr resultados 
en el aspecto motor y técnico en la práctica de los fundamentos técnicos del futbol con balón y sin balón. Además, el niño de acuerdo a su edad adquiere mejoras en sus habilidades motrices básicas, en esto incide su interacción con su contexto, donde vive, con que familiares y amigos se relaciona, desarrolla instintivamente estas cualidades (Dos Santos \& Gonzales, 2013; Haywood \& Getchell, 2014). Entonces se nota el incremento en forma progresiva de su patrón motor, es decir su actividad motriz adquiere cambios y esto repercute en su madurez, logrando mejoras en sus habilidades motrices para luego incrementar sus destrezas motrices. (Madrona, Contreras y Barreto, 2008; Martinez, 2000). Las habilidades motoras se pueden categorizar en función de los ámbitos donde se desarrollan o desenvuelven los niños, lo que permite y facilita la coordinación motriz (Alvarez \& Pazos, 2020; Palmar, 2014). Además, desarrolla en el niño estas capacidades, mediante las funciones de Locomoción, manipulación y estabilidad. (Batalla 2000; Castañer \& Camerino, 2006; Fort, 2016; Guevara 2017), posterior a ello mejora sus habilidades motrices específicas y estos se dan a conocer en la práctica de los fundamentos técnicos de los deportes.

Las habilidades locomotrices permiten el desplazamiento del sistema corporal, estos mejoran con la práctica permanente que realizan los niños de acuerdo a su contexto, es decir donde se relaciona, con sus familiares, con sus amigos, si procede de familiares deportistas, gracias a esto el niño desarrolla progresivamente estas habilidades como son: caminar, correr, saltar, galopar, subir, bajar, trepar, rodar. (Araujo, 2012; Batalla 2000; Castañer \& Camerino, 2006; Fort, 2016; Guevara 2017),

Cuando nos referimos a las habilidades manipulativas estas permiten al niño desarrollar sus capacidades respecto a su precisión, que cada vez se incrementa en la adquisición de movimientos motrices, es decir mejora su dominio de destrezas específicas de los deportes que practica, por ejemplo: el fútbol, voleibol, básquetbol, etc. Que permitan a este cada vez mejores lanzamientos, recepciones y botes. (Alarcón, 2008; Peraza, et al. 2020). (Costa Riveiro, 2018; Krebs, et al. 2010). Además, se adquieren por herencia biológica y cultural, de esta manera desarrollaran un repertorio de capacidades coordinativas como: lanzar, recepcionar, golpear, atrapar, driblar, batear. Castañer y Camerino (1991) (Gallahue, 2012; Paine, 2012). Batalla, (2006). Gallague, (2012); Nazario, (2014). En el desarrollo del fútbol estas habilidades motrices se perfeccionan con la práctica de estos, es decir el futbolista va 
mejorando las habilidades manipulativas como recepcionar el balón, rematar a portería, lanzar un pase largo, driblar al adversario para prosperar en ataque.

Por último, las habilidades de estabilidad se clasifican en: empujar, arrastrarse, colgarse, equilibrarse, levantarse, estirarse, doblarse, girar. (Fort, 2016; Díaz 1999). En esa perspectiva estas acciones motrices son elementales en el desarrollo motor de los niños, contrariamente con los hallazgos de la presente investigación realizados con los niños de las instituciones educativas de Puno cercado presentan dificultades y limitaciones en los ítems relacionados a las habilidades de estabilidad, por lo tanto, se sugiere poner mayor énfasis en cada uno de estos postulados. (Leyhr, et el. 2018; Peraza, 2020; Gonzales 2019).

Los fundamentos técnicos del fútbol vienen a ser movimientos que realiza el futbolista cuando conduce, realiza controles orientados, variedad de carreras con el único fin de trasladarlo al marco contrario y anotar un gol. La técnica debe estar orientado al control, dominio y demás acciones técnicas con balón y sin balón en el fútbol (Bauer 1992; Deprez, 2015; Serrano et al. 2017). En ese marco las capacidades técnicas del fútbol, según ESEFUL (2016). Son jugadas sucesivas que se desarrollan coordinadamente y van enlazadas entre sí, se da en el trascurso de un partido de fútbol, se perfeccionan en los entrenamientos y estos son: los diversos tipos de pases, la variedad en la conducción, el dribling, remate con los diversos segmentos del cuerpo en especial los pies, la recepción con los diversos segmentos del cuerpo entre ellos tenemos recepción con el pecho, muslo, empeine, cabeza, hombro, etc. (Ivan Jukik. 2019; Kosiel y Tufano, 2018). Son considerados como fundamentos técnicos sin balón la finta, carreras con cambios de dirección, los saltos. Entonces la finta según (Honer, 2017; Kookstejn, Musalek, 2019) consiste en realizar una sucesión de movimientos con el fin de engañar, desorientar, eludir al adversario. En síntesis, es engañar al adversario con un movimiento del cuerpo para salir por el otro lado. Carreras con cambio de dirección, en el fútbol moderno es un principio táctico ofensivo, se puede realizar ya sea en línea recta, en zigzag, recorrer distancias pequeñas de 5, 10, 15, 20 metros. (Honer, 2017; Read 2018; Unnithan, 2012). Finalmente tenemos los saltos en el fútbol son esenciales, se dan en todas las acciones del encuentro tanto en defensa como en ataque. Los saltos se mejoran saltando, es decir el tren inferior adquiere mayor fuerza y potencia. (Gullich 2014; Valentedos-Santos 2012).

\section{MÉTODOS}


El tipo de investigación es básica o pura. investigación básica o pura porque parte de un marco teórico y se basa en el para crear nuevas teorías determinados por las variables: habilidades motrices básicas y los fundamentos técnicos del fútbol; y además es de tipo explicativo, porque explica la consecuencia de la variación de las variables en estudio. (Hernández, Fernández y Baptista, 2016). El diseño es correlacional causal, porque describen las relaciones de causalidad entre las variables habilidades motrices básicas y fundamentos técnicos del fútbol. (Hernández, Fernández y Baptista, 2016).

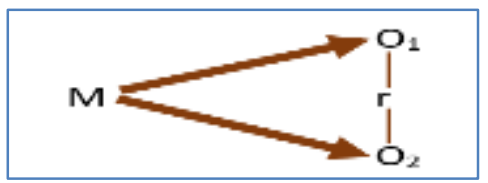

$\mathbf{X}_{1}$ : Habilidades motrices básicas

$\mathbf{X}_{2}$ : Fundamentos técnicos del fútbol

\section{RESULTADOS}

Tabla 1: Resultados de los fundamentos técnicos del fútbol, en niños de 9 a 11 años de las instituciones educativas primarias de la ciudad de Puno

\begin{tabular}{|c|c|c|c|c|c|c|c|c|c|c|}
\hline \multirow{2}{*}{$\begin{array}{c}\text { Nivel de } \\
\text { fundamentos } \\
\text { técnicos del } \\
\text { fútbol }\end{array}$} & \multicolumn{2}{|c|}{ Muy Bueno } & \multicolumn{2}{|c|}{ Bueno } & \multicolumn{2}{|c|}{ Deficiente } & \multicolumn{2}{|c|}{$\begin{array}{c}\text { Muy } \\
\text { Deficiente }\end{array}$} & \multicolumn{2}{|c|}{ TOTAL } \\
\hline & Frec. & Porc. & Frec. & Porc. & Frec. & Porc. & Frec. & Porc. & Frec. & Porc. \\
\hline $\begin{array}{l}\text { Fundamentos } \\
\text { técnicos del } \\
\text { fútbol con } \\
\text { balón }\end{array}$ & 1,1 & $1,4 \%$ & 52,4 & $64,7 \%$ & 27,0 & $33,3 \%$ & 0,5 & $0,6 \%$ & 81 & $100,0 \%$ \\
\hline $\begin{array}{l}\text { Fundamentos } \\
\text { técnicos del } \\
\text { fútbol sin } \\
\text { balón }\end{array}$ & 0,7 & $0,8 \%$ & 55,3 & $68,3 \%$ & 25,0 & $30,9 \%$ & 0,0 & $0,0 \%$ & 81 & $100,0 \%$ \\
\hline TOTAL & $\mathbf{0 , 9}$ & $1,1 \%$ & 53,9 & $66,5 \%$ & 26,0 & $32,1 \%$ & 0,3 & $0,3 \%$ & 81,0 & $100,0 \%$ \\
\hline
\end{tabular}

Fuente: Cuadros del $\mathrm{N}^{\circ} 6$ al $\mathrm{N}^{\circ} 8$

Elaboración: El ejecutor

De la tabla 1 , se puede deducir que después de haber aplicado el instrumento de la variable 2 Fundamentos técnicos del fútbol en actividades con balón y sin balón, tomados a los 81 estudiantes de las instituciones educativas primarias de Puno en el año 2019, se tiene que el $66.5 \%$ está en el nivel bueno, mientras que el $1.1 \%$ se encuentra en la categoría de muy 
bueno, seguido de un $32.1 \%$ se encuentra en la categoría deficiente, mientras que solo el $0.3 \%$ está en la categoría muy deficiente. Queda demostrado según nuestros resultados y en comparación con los antecedentes de la presente investigación de que el niño mejora su capacidad técnica en los fundamentos técnicos del fútbol en actividades con balón y sin balón como plantean en sus investigaciones Solorzano (2017); Ivan Jukic (2019).

Tabla 2: Resultados del nivel habilidades motrices en niños de 9 a 11 años de las instituciones educativas primarias de la ciudad de Puno

\begin{tabular}{c|cc|cc|cc|cc|cc}
\hline $\begin{array}{c}\text { Nivel de } \\
\text { habilidades } \\
\text { motrices } \\
\text { básicas }\end{array}$ & \multicolumn{2}{|c|}{ Muy Bueno } & \multicolumn{2}{|c|}{ Bueno } & Deficiente & \multicolumn{2}{c|}{$\begin{array}{c}\text { Muy } \\
\text { Deficiente }\end{array}$} & \multicolumn{2}{|c}{ TOTAL } \\
\cline { 2 - 10 } & Frec. & Porc. & Frec. & Porc. & Frec. & Porc. & Frec. & Porc. & Frec. & Porc. \\
\hline Locomoción & 1,9 & $2,3 \%$ & 55,8 & $68,8 \%$ & 23,1 & $28,5 \%$ & 0,3 & $0,3 \%$ & 81 & $100,0 \%$ \\
\hline Manipulación & 4,5 & $5,6 \%$ & 57,5 & $71,0 \%$ & 19,0 & $23,5 \%$ & 0,0 & $0,0 \%$ & 81 & $100,0 \%$ \\
\hline Estabilidad & 6,5 & $8,0 \%$ & 59,3 & $73,1 \%$ & 15,1 & $18,7 \%$ & 0,1 & $0,2 \%$ & 81 & $100,0 \%$ \\
\hline TOTAL & $\mathbf{4 , 3}$ & $5,3 \%$ & $\mathbf{5 7 , 5}$ & $71,0 \%$ & $\mathbf{1 9 , 1}$ & $23,6 \%$ & $\mathbf{0 , 1}$ & $0,2 \%$ & $\mathbf{8 1 , 0}$ & $100,0 \%$ \\
\hline
\end{tabular}

Fuente: Cuadros del $\mathrm{N}^{\circ} 3$ al $\mathrm{N}^{\mathrm{o}} 5$.

Elaboración: El ejecutor

De la tabla 2, se puede deducir que después de haber aplicado el instrumento de la variable 1 Habilidades motrices básicas, específicamente en la dimensión 1 nivel de las habilidades de estabilidad, dimensión 2, habilidades de locomoción, dimensión 3, habilidades de estabilidad a los 81 estudiantes de las instituciones educativas primarias de Puno en el año 2019, se tiene que el 71.0\% está en el nivel bueno, mientras que el 5.3\% se encuentra en la categoría de muy bueno, seguido de un $23.6 \%$ que se ubican en la categoría de deficiente y solo el $0.2 \%$ se encuentran en la categoría de muy deficiente. Queda demostrado según nuestros hallazgos cuando apostamos por mejorar las capacidades coordinativas el niño mejora su desenvolvimiento motriz, el cual permite compartir y valorar estudios propuestos por Castañer y Camerino (1991); Hart (2016); Gallague (2006). 
Tabla 3: Correlación entre las variables habilidades motrices y los fundamentos técnicos del Fútbol en niños de instituciones educativas de primaria Puno 2019

\section{Correlaciones}

\begin{tabular}{|ll|c|c|}
\hline & Correlación de Pearson & $\begin{array}{c}\text { Habilidades } \\
\text { Motrices }\end{array}$ & $\begin{array}{c}\text { Fundamentos } \\
\text { Técnicos de } \\
\text { Fútbol }\end{array}$ \\
\hline Habilidades Motrices & Sig. (bilateral) & 1 &, $681^{* *}$ \\
& $\mathrm{~N}$ & 81 &, 000 \\
Fundamentos Técnicos & Correlación de Pearson &, $681^{* *}$ & 81 \\
de Fútbol & Sig. (bilateral) &, 000 & 1 \\
& $\mathrm{~N}$ & 81 & 81 \\
\hline
\end{tabular}

**. La correlación es significativa al nivel 0,01 (bilateral).

De la tabla 3, se precisa los resultados según el SPSS igual a $0.681 * *$ nos indica que existe una correlación altamente significativa entre las habilidades motrices básicas y los fundamentos técnicos de fútbol en niños de 09 y 11 años de las instituciones educativas de educación primaria de Puno, en el año 2019. Nuestros hallazgos nos permiten demostrar que las habilidades motrices tienen relevancia y mejoran progresivamente el nivel de los fundamentos técnicos del fútbol. ESEFUL (2016), del mismo modo tenemos otras investigaciones que llegan a conclusiones similares como los expuestos por Jakub Kokstejn, et al. (2019) consideran que las capacidades motoras y físicas influyen en los futbolistas menores.

Tabla 4: Prueba de Normalidad de Habilidades Motrices Básicas y Fundamentos Técnicos Fútbol

\begin{tabular}{lc|c|c}
\hline & \multicolumn{3}{c}{ Kolmogorov-Smirnov $^{\mathbf{a}}$} \\
\cline { 2 - 4 } & Estadístico & gl & Sig. \\
\hline LOC &, 116 & 81 &, 009 \\
\hline MAN &, 133 & 81 &, 001 \\
\hline EST &, 159 & 81 &, 000 \\
\hline HMB &, 099 & 81 &, 046 \\
\hline FCB &, 145 & 81 &, 000 \\
\hline FSB &, 149 & 81 &, 000 \\
\hline FTF &, 104 & 81 &, 031 \\
\hline
\end{tabular}

Note. Data de Habilidades Motrices Básicas y Fundamentos Técnicos Fútbol 


\section{Interpretación:}

Todos los Sig. de Habilidades Motrices Básicas y Fundamentos Técnicos Fútbol y sus Dimensiones son $<0.05$, por tanto, la distribución de sus datos es No Paramétrica, y para la relación se debe de usar la fórmula de Rho Spearman.

\section{DISCUSIÓN}

La relevancia de la presente investigación radica en la importancia de las habilidades motrices básicas en la formación del futuro futbolista, porque mediante estas habilidades el niño mejora progresivamente su desarrollo motor (Fort, 2016; Gallague 2006) por consiguiente sus habilidades específicas, en este caso sus capacidades técnicas en lo que respecta a los fundamentos técnicos del fútbol Costa Riveiro, (2018). Ivan Jukig (2019). La propuesta desarrollada por Jakub Kookstejn, Martin Musalek (2019) se relaciona con las dos variables en estudio porque afirman que las habilidades motrices básicas como las manipulativas, locomotrices y de estabilidad influyen en las habilidades específicas, en este caso en el deporte del fútbol, es decir en los fundamentos técnicos del fútbol, en estrecha relación con las capacidades físicas básicas e iniciación en las capacidades tácticas del fútbol, realizado en la Universidad de CZECH REPUBLIC. Por consiguiente, este estudio resalta nuestros resultados estadísticos. Tomando como referencia los autores mencionados según mi experiencia profesional con estudiantes de la región de Puno puedo resaltar que con los resultados de la investigación realizada contribuyo a que el deporte del fútbol en edades inferiores alcance un desarrollo técnico progresivo porque con una adecuada planificación y disciplina en el trabajo esto tiene que cambiar, dejando de lado el pensamiento negativo, el trabajo sin objetivos, porque la práctica del fútbol implica trabajo, disciplina y perseverancia. ESEFUL (2016). Honer, 2017; Kookstejn, 2019; Musalek, 2019; Serrano, 2017).

Del mismo modo Solorzano, (2017) concluye que el niño, el púber de acuerdo como va desarrollando sus destrezas coordinativas, estas a su vez mejoran sus habilidades específicas en el fútbol, los cuales se manifiestan en su desenvolvimiento con el balón, mejora su riqueza técnica, su forma de correr, su postura en el campo, su equilibrio cuando va al choque o en la disputa de un balón dividido. El estudio lo realizó con niños de la categoría sub 12, de la I.E.P. N N $^{\mathrm{a}} 21544$ La Villa, realizado en la Universidad Nacional José Faustino Sanchez Carrión de Huacho Lima, el cual refuerza y orienta la presente investigación porque ambos trabajos se realizaron con menores de edad, a diferencia del estudio menciona el aporte 
nuestro es mayor en el sentido que nuestra propuesta abarca a todas las instituciones educativas primarias del cercado de Puno.

Según las variables en estudio como son: habilidades motrices básicas (Batalla 2000; Castañer \& Camerino, 2006; Fort, 2016; Guevara 2017; Peraza, et al., 2020), y fundamentos técnicos del futbol el niño aprende a través de la observación, imitación, mediante juegos, por tal razón es fundamental el uso correcto de materiales variados como escaleras de coordinación, balones de fútbol, conos, platillos, estacas, cintas para demarcar terreno, aros, vallas pequeñas para multisaltos, campos deportivos con césped, campos deportivos de fútbol sintético, chalecos, etc. Con todas estas condiciones y empleando métodos, estrategias metodológicas pertinentes el niño mejora progresivamente sus capacidades motoras, capacidades físicas y sus capacidades técnicas. (Blásquez 2006; Smits, et al., 2020; Serrano et al., 2017). Los resultados de la investigación demuestran que las actividades locomotoras, manipulativas y de estabilidad en los estudiantes en estudio nos demuestran que la mayoría se encuentra en la categoría bueno, tomando en cuenta las instituciones educativas del cercado de Puno, en contra posición con antecedentes que plantea Alonso, (2020) concluye que se debe mejorar la motricidad porque una gran mayoría no trabaja eficientemente, tomando como referencia a las aulas de los infantes materia de su investigación realizada. Costa Riveiro, (2018) en su estudio realizado en niños llegó a la conclusión de que gracias a la práctica deportiva orientada el niño mejora progresivamente su desarrollo motor, dicho hallazgo refuerza nuestros resultados en el sentido que ambos llegamos a conclusiones similares.

Como podemos observar los resultados de estas actividades referentes a los fundamentos técnicos del fútbol en la mayoría de los estudiantes de las instituciones educativas de Puno cercado se encuentran en la categoría de bueno, tanto en las actividades con balón y sin balón, Refuerzan esta variable el antecedente expuesto por Ivan Jukic (2019) donde aclara y señala que en la formación de futbolistas con condiciones es fundamental mejorar sus habilidades motoras y sus capacidades técnicas. sus resultados fortalecen nuestra investigación porque ambos llegamos a la conclusión de que el niño con la práctica disciplinada mejora su desenvolvimiento en su accionar con el balón y por consiguiente en el campo de juego.

En comparación con los resultados obtenidos por Ivan Jukic (2019) donde realiza pruebas subjetivas de las habilidades y pruebas objetivas de la técnica y la motricidad de los niños, 
los cuales demuestran que los usaría para seleccionar deportistas que reúnan estas cualidades $\mathrm{y}$ de esta forma lograr deportistas competitivos. Corrobora con estos principios Jakub Kokstejn. et al., (2019) al concluir que las habilidades motoras mejoran el rendimiento de sus habilidades específicas relacionadas a la técnica del fútbol en lo que respecta al pase, recepción, dribling, saque lateral, juego de cabeza, remate, conducción en los niños, los cuales influyen en los futbolistas menores en su riqueza técnica con el balón.

Por otro lado según los resultados de la presente investigación, cuando las habilidades motoras básicas se trabajan en los niños, estos mejoran y para ello tiene influencia su grupo etario, el aspecto social, la convivencia con niños los cuales comparten ese ambiente de confianza, razón por la cual se incrementa el desarrollo de su condición motora, asimismo cuando incrementamos la práctica del fútbol, en este caso insertamos el balón de fútbol, trabajando siguiendo una secuencia técnica, el uso adecuado del material, metodologías pertinentes de acuerdo a su edad, se observa el crecimiento de sus capacidades técnicas, mejora su condición física y estos se encuentran expeditos para la iniciación de sus capacidades tácticas, se demostró que los niños de la ciudad de Puno se encuentran en la categoría de bueno y muy bueno tanto en sus habilidades motoras como en el dominio de los fundamentos técnicos del fútbol. Analizando otros estudios a nivel nacional e internacional entre ellos citamos a (Ivan Jukic 2019; Solorzano, 2017), quienes llegaron a resultados similares en su aspecto locomotor, del mismo modo en la mejora de sus cualidades técnicas con el balón de fútbol, es por eso que es pertinente desde instancias como el MINEDU, IPD, Gobiernos Regionales, Direcciones Regionales de Educación, UGELs, Instituciones Educativas a lo largo y ancho del país trabajar las habilidades motrices básicas en el fútbol, para lo cual el docente de educación física tiene que estar preparado para brindar conocimiento teórico y práctico relacionado a la materia en estudio.

Las habilidades motrices y el deporte del fútbol en nuestra ciudad de Puno en edades tempranas es escasa, hace falta un trabajo de base, a largo plazo, el error que se comete no solamente en la región Puno sino en todo el país, es que se realiza una planificación a corto plazo, en el cual no se ve un trabajo real, motivo por el cual se abandona proyectos, se abandona a estudiantes deportistas que tienen talento para el fútbol. (Leyhr, et al. 2018; Peraza; 2020). 


\section{CONCLUSIONES}

Con los resultados de la Prueba de Hipótesis general haciendo uso de la correlación de Pearson podemos determinar que las habilidades motrices básicas influyen en los fundamentos técnicos del fútbol porque los resultados según el SPSS igual a $0.681^{* *}$ nos indica que existe una correlación altamente significativa entre las habilidades motrices básicas y los fundamentos técnicos de fútbol en niños de 09 y 11 años de las instituciones educativas de educación primaria de Puno, en el año 2019.

Según los resultados de la investigación podemos establecer que las habilidades motrices básicas influyen en los fundamentos técnicos del fútbol con balón en niños de 09 y 11 años de las instituciones educativas de educación primaria de Puno, en el año 2019, dado que la prueba de correlación de Pearson nos muestra un valor de igual a $0.662 * *$ el que nos indica que existe una correlación altamente significativa entre las habilidades motrices básicas y los fundamentos técnicos del fútbol con balón, además podemos determinar que la dimensión de las habilidades motrices con mayor relación con los fundamentos técnicos del fútbol con balón es la locomoción.

Con resultados de la investigación presentadas en tablas y figuras podemos establecer que las habilidades motrices básicas influyen en los fundamentos técnicos del fútbol sin balón en niños de 09 y 11 años de las instituciones educativas de educación primaria de Puno, en el año 2019, dado que la prueba de correlación de Pearson nos muestra un valor de igual a $0.645^{* *}$ el que nos indica que existe una correlación altamente significativa entre las habilidades motrices básicas y los fundamentos técnicos del fútbol sin balón, , además podemos determinar que la dimensión de las habilidades motrices con mayor relación con los fundamentos técnicos del fútbol sin balón es la locomoción.

\section{REFERENCIAS}

Alonso, Y. (2020) Importancia percibida de la motricidad en educación infantil en los centros educativos de Vigo. España.

Álvarez y Pazos. (2020) Importancia percibida de la motricidad en educación infantil en los centros educativos de Vigo. España.

Araújo MP, Barela JA, Celestino ML, Barela AMF. 2012; Contribución de diferentes contenidos de las clases docentes Educación Primaria Educación Física para el desarrollo de las habilidades motoras fundamentales. Rev Bras Med Esporte. DOI: 10.1590 / S1517-86922012000300002 
Batalla A. 2006. Habilidades motrices. Barcelona: INDE; España.

Castañer y Camerino (1991) Habilidades motrices básicas

Costa Riveiro, P. et. al (2018). Desempeño motor en habilidades básicas de niños participantes y no participantes de práctica deportiva orientada. Universidade Federal de Minas Gerais, Belo Horizonte-MG, Brasil.

Deprez, D., Valente-Dos-Santos, J., Coelho-e-Silva, M. J., Lenoir, M., Philippaerts, R., \& Vaeyens, R. (2015a). Longitudinal development of explosive leg power from childhood to adulthood in soccer players. International journal of sports medicine, 36(08), 672-679.

Díaz, L. J. (1999). La enseñanza y aprendizaje de las habilidades y destrezas motrices básicas. Colección la educación física en reforma.INDE. Barcelona: INDE. Publicaciones. España.

ESEFUL, (2016) Módulos de capacitación: Carrera de Directores Técnicos de Fútbol. Lima.

Flores, B. (2000). Habilidades motrices. Barcelona: INDE Publicaciones. España.

Fort, A. (2016) ¿Por qué es importante desarrollar la competencia motriz en la infancia y la adolescencia? Base para una vida saludable. Barcelona España,

Gallahue, D. L., Ozmun, J. C., \& Goodway, J. (2011). Understanding motor development: Infants, children, adolescents, adults. (7th ed.). New York, NY: McGraw-Hil.

Garay y Romero (2012), El juego como medio didáctico en el desarrollo de las habilidades motrices básicas de los alumnos del IV ciclo del nivel primario de la I.E.P. "La edad de oro”. Universidad Nacional José Faustino Sánchez Carrión de Huacho. Lima.

Gonzales, R. (2019) Actividades físicas para desarrollar las habilidades motrices básicas en niños del programa educa a tu hijo. Universidad de Cienfuegos "Carlos Rafael Rodriguez". Cuba.

Guevara, P, (2017) La técnica de carrera y el desarrollo motriz en aspirantes a soldados. Universidad de las fuerzas armadas ESPE. Ecuador.

Gullich, A. 2014. Selection, de-selection and progression in German football talent promotion. Eur. J. Sport Sci. [CrossRef] [PubMed]

Hernández, Fernández y Baptista. (2014). Metodología de la investigación. 6ta. Edición, Editorial Edamsa Impresiones, S.A. de C.V. México.

Höner, O., Leyhr, D., and Kelava, A. (2017). The influence of speed abilities and technical skills in early adolescence on adult success in soccer: a longterm prospective analysis 
using ANOVA and SEM approaches. PLoS One 12:e0182211. doi: 10.1371/journal.pone.0182211

Ivan Jukik (2019) Identificación de diferencias en los niveles de rendimiento de U10 jugadores de fútbol. Universidad de Nueva Zelanada. Nueva Zelanda.

Kokstejn, J., Musalek, M., and Tufano, J. J. (2017b). Are sex differences in fundamental motor skills uniform throughout the entire preschool period? PLoS One 12:e0176556. doi: 10.1371/journal.pone.0176556

Kozieł, S. M., and Malina, R. M. (2018). Modified maturity offset prediction equations: validation in independent longitudinal samples of boys and girls. Sports Med. 48, 221-236. doi: 10.1007/s40279-017-0750-y

Leyhr, D., Kelava, A., Raabe, J., and Höner, O. (2018). Longitudinal motor performance development in early adolescence and its relationship to adult success: an 8-year prospective study of highly talented soccer players. PLoS One 13:e0196324. doi: 10.1371/journal.pone.0196324

Madora, P. (2008). Habilidades motrices en la infancia y su desarrollo desde una educación física animada. Revista Iberoamericana de educación. Madrid España.

Martinez, Mujica. (2006). Programa de juegos deportivos. Investigación de campo.

Nazário PF, Vieira JLL. 2014 Contexto deportivo y desarrollo motor del niño. Rev Bras Cineantropom Hum Performance. DOI: 10.5007 / 1980-0037.2014v16n1p86

Naupas. H. (2018). Metodología de la investigación cuantitativa - cualitativa y redacción de la tesis. Editorial AAdriana Gutierrez M. Ediciones de la U. Bogota Colombia.

Paine V, Isaacs L. 2001. Human motor development: A lifespanapproach. 5th ed. New York: McGraw-Hill.

Peraza, C. (2020) Medio de enseñanza para el desarrollo de habilidades motrices básicas en niños de sexto año de vida. Universidad "Jesús Montane Oropesa" Isla de la Juventud. Cuba.

Read, P.J.; Jimenez, P.; Oliver, J.L.; Lloyd, R.S. 2018. Injury prevention in male youth soccer: Current practices and perceptions of practitioners working at elite English academies. J. Sports Sci. [CrossRef]

Serrano, J. S., Pizarro, A. P., García-González, L., Domínguez, A. M., and Álvarez, F. V. (2017). Evolution of tactical behavior of soccer players across their development. Int. J. Perform. Anal. Sport 17, 885-901. 
Solorzano, (2017), Las capacidades coordinativas y los fundamentos técnicos del fútbol en la categoría sub 12 de la institución educativa $\mathrm{N}^{\circ} 21544 \mathrm{La}$ Villa. Universidad Nacional José Faustino Sanchez Carrión. Huacho Lima.

Tafur, P. R. (2014), como hacer un proyecto de investigación. Lima: Editorial Gráfica Educativa. Perú.

Unnithan, V., White, J., Georgiou, A., Iga, J., and Drust, B. (2012). Talent identification in youth soccer. J. Sports Sci. 30, 1719-1726. doi: 10.1080/02640414.2012.731515 